\title{
Coherent Beam Splitting by a Thin Grating
}

\author{
M. Božić $\tilde{C}^{a, *}$, D. DimiĆ ${ }^{b}$ AND M. DavidoviĆc \\ ${ }^{a}$ Institute of Physics, University of Belgrade, Serbia \\ ${ }^{b}$ Faculty of Mathematics and Natural Sciences, Niš, Serbia \\ ${ }^{c}$ Faculty of Civil Engineering, University of Belgrade, Serbia
}

\begin{abstract}
The wide use of beam splitters in quantum interferometry, attosecond metrology, modern quantum information processing, foundations of quantum mechanics, has been based on coherence of beams emerging from a beam splitter. Here we further develop the approach in which a beam splitter is regarded as a transformer of an incident wave field (photon field or matter wave field) into a field which has narrow maxima at the points along and in close vicinity of two or more particular lines. This description was derived by considering a thin grating as a model of a beam splitter for photons, atoms and molecules. It is applied to answer some questions raised in the discussions on the interpretation of wave particle duality.
\end{abstract}

PACS numbers: 03.75.--b, 03.75.Be, 03.75.Dg, 42.25.Fx, 42.25.Hz

\section{Introduction}

Beam splitter is a device which is in the heart of quantum interferometry [1], attosecond metrology [2], modern quantum information processing [3], the debate on the foundations of quantum mechanics and interpretation of a wave function $[1,4,5]$. But, a beam splitter's name reflects only one of its essential properties: that it splits an initial beam into two beams. This name does not take into account either the mutual coherence of the two beams, which it generates from an initial narrow beam, nor the influence of this coherence on a particle associated with a wave. This name neither reflects its property to entangle two initial coherent beams [6]. On the other hand, all these properties are crucial for the above mentioned wide application of beam splitters. Maybe it would be useful to give a name "coherent beam splitter" to a beam splitter which performs coherent beam splitting. Here we present a theoretical description of beam splitting by a thin grating which justifies this proposal. The results of a dynamic theory of diffraction, on which a neutron beam splitter $[1,7]$ is based could be represented in an analogous way.

\section{Transformation of an incident wave by a thin grating}

The wave function of a massive particle behind a grating is a solution of the time-dependent free particle Schrödinger equation associated with the wave function incident to a grating and modified by this grating. This modification is taken into account through boundary conditions, usually expressed using the transmission function $T(x)$. For simplicity, we shall consider a one-

\footnotetext{
* corresponding author; e-mail: bozic@ipb.ac.rs
}

-dimensional grating (Fig. 1). The incident wave function is assumed, in general, to have the form:

$$
\begin{aligned}
& \Psi^{\mathrm{inc}}(x, y, t)=\mathrm{e}^{-\mathrm{i} \omega t} \mathrm{e}^{\mathrm{i} y k_{y, 1}} \mathrm{e}^{\mathrm{i} x k_{x, 1}} W(x) \\
& +q \mathrm{e}^{-\mathrm{i} \omega t} \mathrm{e}^{\mathrm{i} \varphi} \mathrm{e}^{\mathrm{i} y k_{y, 2}} \mathrm{e}^{\mathrm{i} x k_{x, 2}} W(x), \quad y \leq 0,
\end{aligned}
$$

where $k_{x, 1}^{2}+k_{y, 1}^{2}=k_{x, 2}^{2}+k_{y, 2}^{2}=k^{2}, k_{x, 1} \ll k_{y, 1} \approx k$, $k_{x, 2} \ll k_{y, 2} \approx k, \omega=k c$. The quantities $\varphi$ and $q$ are constants and $W(x)$ is a real function which describes the extent along the $x$ axis of each plane wave component of the incident wave. For $q \neq 0$ function $\Psi^{\mathrm{inc}}$ is a superposition of two plane waves, for $q=0$ it reduces to a plane wave. The evolution of wave (1) behind a grating is given by

$$
\Psi(x, y, t)=B \mathrm{e}^{\mathrm{i} k y} \mathrm{e}^{-\mathrm{i} \omega t} \psi(x, t),
$$

where

$$
\psi(x, t)=\frac{1}{\sqrt{2 \pi}} \int_{-\infty}^{\infty} \mathrm{d} k_{x} c\left(k_{x}\right) \mathrm{e}^{\mathrm{i} k_{x} x} \mathrm{e}^{-\mathrm{i} \hbar k_{x}^{2} t / 2 m}
$$

is the transverse wave function, $B$ is the normalization constant and $c\left(k_{x}\right)$ is the probability amplitude of particle transverse momentum (in $\hbar$ units), or the transverse wave function in the momentum representation [8]. Function $c\left(k_{x}\right)$ is determined by the incident function at the point $y=0^{-}$and the properties of the grating. If the incident wave function has the form as in Eq. (1) the function $c\left(k_{x}\right)$ is a sum of two terms associated with the two terms in $\Psi^{\text {inc }}(x, y, t)$ :

$$
c\left(k_{x}\right)=c_{k_{x, 1}}\left(k_{x}\right)+q \mathrm{e}^{\mathrm{i} \varphi} c_{k_{x, 2}}\left(k_{x}\right) .
$$

The function $c\left(k_{x}\right)$ is very useful for understanding $[8,9]$ the space distribution of particles in the far field because the function $\psi(x, t)$ at a fixed distance $y$ far from the grating is approximated by:

$$
\psi(x, t)=\sqrt{\frac{m}{\hbar t}} \mathrm{e}^{-\mathrm{i} \pi / 4} \mathrm{e}^{\mathrm{i} x^{2} m / 2 \hbar t} c\left(x \frac{m}{\hbar t}\right)
$$

To be applicable to photons, the latter equation should be modified by using the substitution, $t \rightarrow y$ at the left- 

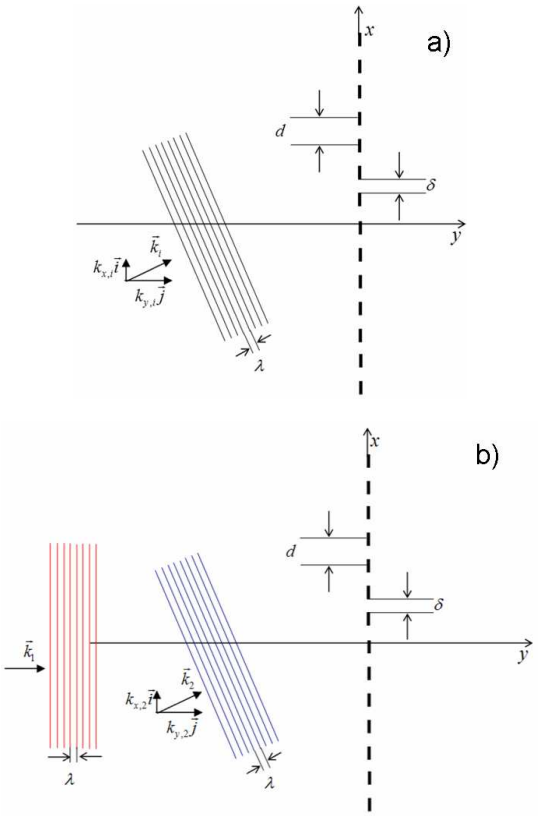

Fig. 1. Free standing grating illuminated by a plane wave (a) and by a superposition of two plane waves (b).

-hand side and $m / \hbar t \rightarrow k / y$ at the right-hand side [9]. Therefore, one gets

$$
\psi(x, y)=\sqrt{\frac{k}{y}} \mathrm{e}^{-\mathrm{i} \pi / 4} \mathrm{e}^{\mathrm{i} x^{2} k / 2 y} c\left(x \frac{k}{y}\right)
$$

The splitting of neutron waves [1, 7] and optical [10] waves by crystal plates may be also described using functions (5) and (6), respectively, and the properties of the probability amplitude of transverse momentum, as we shall show in the forthcoming paper.

\section{Properties of the probability amplitude of transverse momentum}

By denoting the transmission function of the grating by $T(x)$ and assuming that the incident wave illuminates $n$ slits whose mutual distance is $d$, i.e. that

$$
\begin{aligned}
& W(x)=M \\
& \quad \text { for } x \in[-((n-1) d / 2)-\delta / 2,((n-1) d / 2)+\delta / 2], \\
& W(x)=0 \\
& \text { for } x \notin[-((n-1) d / 2)-\delta / 2,((n-1) d / 2+\delta / 2],(7)
\end{aligned}
$$
where $M$ is a constant, we obtain

$$
c_{k_{x, i}}\left(k_{x}\right)=\frac{1}{\sqrt{2 \pi}}
$$

$$
\times \int_{-((n-1) d / 2)-\delta / 2}^{+((n-1) d / 2)+\delta / 2} \mathrm{~d} x^{\prime} \mathrm{e}^{\mathrm{i} k_{x, i} x^{\prime}} \mathrm{e}^{-\mathrm{i} k_{x} x^{\prime}} T\left(x^{\prime}\right) .
$$

Assuming that the slits are totally transparent (for all $x$ at a slit $T(x)=1$ ) and the bars totally absorbing (for all $x$ at a bar $T(x)=0$ ), one finds the following expression for the probability amplitude of transverse momentum behind a grating with $n$ slits of width $\delta$ with mutual distance $d$ :

$$
\begin{aligned}
& c_{k_{x, i}}\left(k_{x}\right)=\frac{1}{\sqrt{2 \pi}} \frac{1}{\sqrt{\delta n}} \\
& \quad \times \frac{2 \sin \left(\left(k_{x}-k_{x, i}\right) \delta / 2\right)}{\left(k_{x}-k_{x, i}\right)} \frac{\sin \left(\left(k_{x}-k_{x, i}\right) n d / 2\right)}{\sin \left(\left(k_{x}-k_{x, i}\right) d / 2\right)}
\end{aligned}
$$

In the case of a Ronchi grating, $d=2 \delta$, the above expression reduces to a simpler expression

$$
\begin{gathered}
c_{k_{x, i}}\left(k_{x}\right)=\frac{1}{\sqrt{\pi d n}} \frac{1}{\left(k_{x}-k_{x, i}\right)} \\
\times \frac{\sin \left(\left(k_{x}-k_{x, i}\right) n d / 2\right)}{\cos \left(\left(k_{x}-k_{x, i}\right) d / 4\right)} .
\end{gathered}
$$

The latter function has three pronounced maxima at $k_{x}$ given by

$$
k_{x}=k_{x, i}, \quad k_{x}=k_{x, i} \pm(2 \pi / d)
$$

and a less pronounced maximum at

$$
\begin{aligned}
& k_{x}=k_{x, i} \pm(6 \pi / d), \\
& c_{k_{x, i}}\left(k_{x, i}\right)=(\sqrt{n d} / \sqrt{\pi}) / 2, \\
& c_{k_{x, i}}\left(k_{x, i} \pm 2 \pi / d\right)=(-1)^{n+1}(\sqrt{n d} / \sqrt{\pi}) / \pi, \\
& c_{k_{x, i}}\left(k_{x, i} \pm 6 \pi / d\right)=(-1)^{n}(\sqrt{n d} / \sqrt{\pi}) / 3 \pi .
\end{aligned}
$$

The important characteristic of the Ronchi grating is

$$
c_{k_{x, i}}\left(k_{x, i} \pm 4 \pi / d\right)=0 .
$$

In between the maxima at (11) the function $c_{k_{x, i}}\left(k_{x}\right)$ oscillates with amplitudes of oscillations much smaller than the values in (12). Outside this range, the values of the function $c_{k_{x, i}}\left(k_{x}\right)$ are negligible.

From the above properties of the function $c_{k_{x, i}}\left(k_{x}\right)$ and Eqs. (2) and (3) it follows: If the incident beam (1) contains only one plane wave component $(q=0), 52.59 \%$ of transmitted particles retain the $x$-component of momentum in the close vicinity of $k_{x, i}$ whereas $42.67 \%$ of transmitted particles acquire new value which differs from the initial value by $2 \pi / d$ or by $-2 \pi / d$, approximately.

In interferometry, of particular interest is coherent splitting when $k_{x, i}$ of the incident wave (1), $i=1,2$, take values from the set

$$
S=[0,2 \pi / d,-2 \pi / d] .
$$

The importance of this set comes from the fact that one or two components of the multi-component outgoing wave propagate along the same direction/directions which characterize the incident beam.

But one has to consider individually two important cases: $(\alpha)$ the incident wave (1) has one component $(q=0)$ characterized by $k_{x, 1} \in[0,2 \pi / d,-2 \pi / d]$ and $(\beta)$ the incident wave $(1)$ has two components $(q \neq 0)$ characterized by $\left(k_{x, 1}=0, k_{x, 2}=2 \pi / d\right)$ or $\left(k_{x, 1}=0\right.$, $\left.k_{x, 2}=-2 \pi / d\right)$. 
( $\alpha$ ) In this case, the probability amplitude of transverse momentum behind the beam splitter has three prominent maxima at $k_{x, m}$, one of which coincides with the $x$ component of momentum of the incident beam

$$
\begin{aligned}
& k_{x, 1}=0, \quad k_{x, m} \in[0,2 \pi / d,-2 \pi / d], \\
& k_{x, 1}=2 \pi / d, \quad k_{x, m} \in[0,2 \pi / d, 4 \pi / d], \\
& k_{x, 1}=-2 \pi / d, \quad k_{x, m} \in[0,-2 \pi / d,-4 \pi / d] .
\end{aligned}
$$

One can see that the coherent beam splitting is a phenomenon in which an incident field, matter wave field or photon EM field, having the form of a narrow beam propagating along certain direction is transformed by a grating to a new field which has a more complex structure than the incident one, especially in the vicinity of a grating $[8,11]$. But, far from a grating this structure is simpler. It consists of three prominent maxima along the $x$ axis and many small oscillations which in the far field move together coherently along the $x$ axis. Combined with a motion along the $y$ axis, this results in a joint motion of prominent maxima along three straight lines and many small maxima in between. The three lines are at the same time the lines of the quantum mechanical current of massive particles [12] and of electromagnetic flow lines of photons [11].

( $\beta$ ) When the incident wave function is the sum of two plane waves propagating along two different directions $\left(k_{x, 1}=0, k_{x, 2}=2 \pi / d\right)$ or $\left(k_{x, 1}=0, k_{x, 2}=-2 \pi / d\right)$, the probability amplitude of transverse momentum is given by (4). From this expression one concludes that maxima of $\left|c\left(k_{x}\right)\right|^{2}$ are determined not only by maxima of functions $c_{k_{x, 1}}\left(k_{x}\right)$ and $c_{k_{x, 2}}\left(k_{x}\right)$, but by the parameters $q$ and $\varphi$, as well. In order to investigate the dependence of maxima of the function $\left|c\left(k_{x}\right)\right|^{2}$ on parameters $q$ and $\varphi$, it is useful to find complex values of the function $c\left(k_{x}\right)$ for $k_{x} \in[0,2 \pi / d,-2 \pi / d, 4 \pi / d,-4 \pi / d, 6 \pi / d,-6 \pi / d]$. It is enough to consider the values of the function $c\left(k_{x}\right)$ associated with the pair $\left(k_{x, 1}=0, k_{x, 2}=2 \pi / d\right)$ since the results and conclusions for the pair $\left(k_{x, 1}=0, k_{x, 2}=\right.$ $-2 \pi / d)$ are similar

$$
c\left(k_{x}\right)=c_{0}\left(k_{x}\right)+q \mathrm{e}^{\mathrm{i} \varphi} c_{2 \pi / d}\left(k_{x}\right) .
$$

Using (12) and (13) we find

$$
\begin{aligned}
& c(0)=\frac{1}{2} \sqrt{\frac{n d}{\pi}}\left[1+(-1)^{n+1} q \mathrm{e}^{\mathrm{i} \varphi} \frac{2}{\pi}\right], \\
& c\left(\frac{2 \pi}{d}\right)=\frac{(-1)^{n+1}}{\pi} \sqrt{\frac{n d}{\pi}} \\
& \quad \times\left[1+(-1)^{n+1} q \mathrm{e}^{\mathrm{i} \varphi} \frac{\pi}{2}\right], \\
& c\left(-\frac{2 \pi}{d}\right)=\frac{(-1)^{n+1}}{\pi} \sqrt{\frac{n d}{\pi}} \\
& c\left(\frac{4 \pi}{d}\right)=\frac{(-1)^{n+1}}{\pi} \sqrt{\frac{n d}{\pi}} q \mathrm{e}^{\mathrm{i} \varphi} \\
& c\left(-\frac{4 \pi}{d}\right)=\frac{(-1)^{n}}{3 \pi} \sqrt{\frac{n d}{\pi}} q \mathrm{e}^{\mathrm{i} \varphi},
\end{aligned}
$$

$$
c\left(\frac{6 \pi}{d}\right)=\frac{(-1)^{n}}{3 \pi} \sqrt{\frac{n d}{\pi}} .
$$

By comparing (12) and (17) we see that the result of the action of a grating on the initial distribution of particle's transverse momentum is very different in cases $(\alpha)$ and $(\beta)$. In the case $(\alpha)$ probability distribution depends on the value of the initial momentum along $x$-axis and the grating constant. In the case $(\beta)$ it depends on the two values of the initial momentum along $x$-axis, the grating constant and quantities $q$ and $\varphi$. The dependence of $\left|c\left(k_{x}\right)\right|^{2}$ on latter two quantities is very important. In fact it is the source of the interference phenomena in Michelson and Mach-Zehnder interferometers in which gratings have been used as beam splitters.

\section{Grating as a beam splitter in Michelson's interferometer for attosecond pulses}

Michelson interferometer for the characterization of attosecond pulses described by Goulielmakis et al. [2, 13]. uses a grating as a beam splitter (Fig. 2). Incident monochromatic plane wave which propagates along $y$-axis $\left(k_{x, 1}=0, q=0\right)$ is transformed by a grating to a wave having maxima along three directions (only two directions are shown at Fig. 2). The components associated with the zeroth-order maximum and the first-order maximum are then reflected from two mirrors, M1 and M2. As a result, a coherent wave containing two components $\left(k_{x, 1}=0, k_{x, 2}=-2 \pi / d, q=-2 / \pi\right)$ is arriving to a grating from its right-hand side.

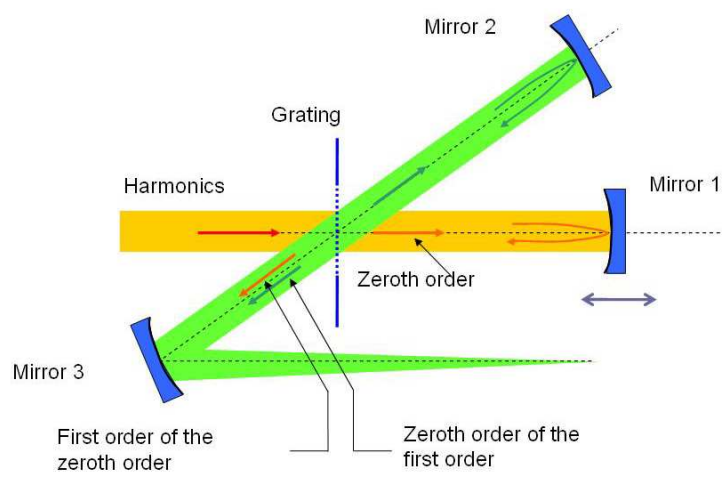

Fig. 2. The scheme of Michelson interferometer with a grating as a beam splitter $[2,13]$.

The wave emerging at the left-hand side of a grating has maxima (components) propagating along several directions. The component propagating in the direction $\left(k_{y}=-k, k_{x}=-2 \pi / d\right)$ has been used in the experiment $[2,13]$. The intensity $I_{3}$ along this direction (towards mirror 3) is determined from Eq. (17b) by substituting $q=-2 / \pi$. It is a periodic function of $\varphi$, which was varied by moving mirror 1 :

$$
I_{3} \propto|c(2 \pi / d)|^{2} \propto 1+(-1)^{n} \cos \varphi .
$$




\section{Summary}

In considering the action of a beam splitter on a quantum particle it is essential to take into account the quantum state of a particle, i.e. its wave function. A time-dependent wave function of a single particle, behind a beam splitter, describes the wholeness of a wave and its evolution $[4,8,9,11]$. The possible paths of a particle are the lines along which its wave function has maxima $[9,11,12]$. This explains the intriguing finding of quantum interferometry, that a single quanton moving along one of the paths has the information about the existence of other paths [1, 14]: this information is due to the wholeness of particle's wave function along and in between these paths.

\section{Acknowledgements}

M. Božić and M. Davidović acknowledge support from the Ministry of Science of Serbia under Project "Quantum and Optical Interferometry", No. 141003.

\section{References}

[1] H. Rauch, S.A. Werner, Neutron Interferometry: Lessons in Experimental Quantum Mechanics Clarendon Press, Oxford 2000, p. 350.

[2] E. Goulielmakis, G. Nersisyan, N.A. Papadogiannis, D. Charalambidis, G.D. Tsakiris, K. Witte, Appl. Phys. B 74, 74 (2002).
[3] Quantum Computation and Quantum Information Theory, C. Macchiavelly, G.M. Palma, A. Zeilinger, World Sci., Singapore 2000.

[4] P. Holland, The Quantum Theory of Motion, Cambridge University Press, Cambridge, UK 1993.

[5] W.P. Schleich, Quantum Optics in Phase Space, Wiley-VCH, Berlin 2001, Ch. 9.

[6] M.S. Kim, W. Son, V. Buzek, P.L. Knight, Phys. Rev. A 65, 032323 (2002).

[7] H. Rauch, D. Petrascheck, in: Neutron Diffraction, Ed. H. Dachs, Springer-Verlag, Heidelberg 1978, p. 303.

[8] D. Arsenović, M. Božić, L. Vušković, J. Opt. B, Quantum Semiclass. Opt 4, S358 (2002).

[9] D. Arsenović, M. Božić, A.S. Sanz, M. Davidović, Phys. Scr. T 134014025 (2009).

[10] M. Born, E. Wolf, Principles of Optics, Cambridge University Press, Cambridge, UK 2002.

[11] M. Davidović, A.S. Sanz, D. Arsenović, M. Božić, S.M. Artes, Phys. Scr. T 135, 014009 (2009).

[12] M. Davidović, D. Arsenović, M. Božić, A.S. Sanz, S. Miret-Artes, Eur. Phys. J. Special Topics 16095 (2008).

[13] http://www.mpq.mpg.de/lpg/research/ attoseconds/attosecond.html .

[14] M. Božić, Z. Marić, Phys. Lett. A 158, 33 (1991). 